\title{
Preliminary studies on the suitability of PETG for 4D printing applications
}

\author{
Mohamed H.Hassan ${ }^{1, *}$, Abdalla M. Omar ${ }^{2}$, Evangelos Daskalakis ${ }^{3}$, Fengyuan Liu $^{4}$, and \\ Paulo Bartolo 5 , \\ ${ }^{1}$ Department of Mechanical, Aerospace and Civil Engineering, University of Manchester. \\ mohamed.hassan@manchester.ac.uk \\ ${ }^{2}$ Department of Mechanical, Aerospace and Civil Engineering, University of Manchester. \\ abdalla.omar@manchester.ac.uk \\ ${ }^{3}$ Department of Mechanical, Aerospace and Civil Engineering, University of Manchester. \\ evangelos.daskalakis@manchester.ac.uk \\ ${ }^{4}$ Department of Mechanical, Aerospace and Civil Engineering, University of Manchester. \\ fengyuan.liu@manchester.ac.uk \\ ${ }^{5}$ Department of Mechanical, Aerospace and Civil Engineering, University of Manchester. \\ paulojorge.dasilvabartolo@manchester.ac.uk
}

\begin{abstract}
Shape memory polymers represent a class of polymers that can recover its original shape under stimulus. This paper presents the preliminary results of a broader research programme that investigates the potential use of polyethylene terephthalate glycol (PETG), a glycol modified version of polyethylene terephthalate (PET), for the fabrication of smart bone tissue engineering scaffolds. PETG. A full characterization analysis is carried out, including chemical analysis, mechanical analysis and shape recovery characteristics. Chemical analysis show the presence of terephthalic acid (TPA), ethylene glycol (EG) and cyclohexanedimethanol (CHDM) in the polymer structure. Mechanical analysis, considering a quasistatic tensile test, shows that PETG presents better than PET. Shape recovery is assessed using a cyclic thermomechanical experiment where stress and temperature are controlled during the programming and recovery phases and demonstrates that PETG is able to change and recover its initial shape.
\end{abstract}

\section{Introduction}

Smart or intelligent materials (e.g. shape memory alloys and polymers, electro-rheological fluids, piezoelectric materials) represent a special class of materials able to change some of their properties in response to changes in their environmental conditions [1]. External stimuli include changes in temperature, pressure, moisture, $\mathrm{pH}$, electrical and magnetic fields or acoustic stimulation $[2,3]$. Due to their potential sensing, actuation, self-cleaning, selfhealing, self-diagnosis and shock absorber properties they are promising materials for a wide range of applications such as construction, aerospace, robotics, biotechnology and tissue engineering $[2,4]$.

\footnotetext{
*Corresponding author: mohamed.hassan@manchester.ac.uk
} 
In the field of tissue engineering, smart materials present significant potential to design smart three-dimensional (3D) biocompatible and biodegradable porous scaffolds (support structures that provide a proper biomechanical environment for cell attachment, differentiation and proliferation, promoting the formation of a new tissue) providing a dynamic change during tissue regeneration and delivering specific stimulations to cells [5]. Scaffolds have been produced using different 3D Printing techniques and a wide range of biocompatible and biodegradable polymers, ceramics and polymer/ceramic composites [6]. The use of 3D Printing to process smart materials, a novel approach known as 4D Printing (3D fabrication of a product that can be programmed to produce a specific response to a stimulus; time and/or functionality is considered as the fourth dimension) [7], is an emerging topic of research in the field of tissue engineering [8]. However, most studies focused on soft active materials and hydrogels and, consequently, limited to scaffolds for non-load bearing application. In order to address this problem, our group is investigating the potential use of polyethylene terephthalate glycol (PETG) for 4D Printing aiming to develop more effective bone scaffolds. Preliminary results are presented in this paper, which investigates the chemical composition, mechanical properties and 4D behaviour of PETG.

\section{Materials and methods}

\subsection{Materials}

PETG with molecular weight of $300 \mathrm{~g} / \mathrm{mol}$ and printing temperature ranging between $195^{\circ} \mathrm{C}$ and $220^{\circ} \mathrm{C}$ was purchased from RS components (UK).

Chloroform (HPLC grade), phenol, and 2, 5 Dihydroxybenzoic acid (DHB), materials required for sample preparation for matrix assisted laser desorption/ionisation time of flight mass spectroscopy (Maldi-ToF-Ms), were purchased from Sigma Aldrich (UK).

\subsection{Fabrication}

PETG dog bone structures were designed using Type I specimen dimensions (Figure 1) according to the ASTM D638-14 standard and produced using a filament-based extrusion 3D printer (Flashforge Creator pro, China) with 100\% infill. Considered processing conditions are shown in Table 1.

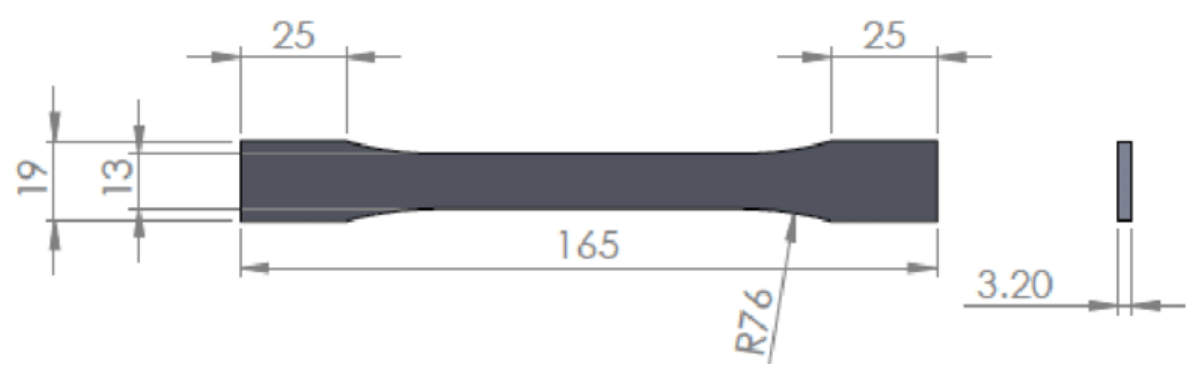

Fig. 1. ASTM D368-14 Type I specimen dimensions

Table 1. Processing conditions

\begin{tabular}{ll}
\hline Parameters & Values \\
\hline Layer thickness $(\mathrm{mm})$ & 0.15 \\
Filament diameter $(\mathrm{mm})$ & 0.40
\end{tabular}




\begin{tabular}{ll} 
Nozzle Temperature $\left({ }^{\circ} \mathrm{C}\right)$ & 230 \\
Bed temperature $\left({ }^{\circ} \mathrm{C}\right)$ & 60 \\
Printing speed $(\mathrm{mm} / \mathrm{s})$ & 60 \\
\hline
\end{tabular}

\subsection{Chemical characterisation}

MALDI-TOF MS, used to characterise the chemical structure of PETG, is based on the use of short, high intensity laser pulses to produce gas ions. In this technique, the analyte molecules are not directly exposed to the laser shots. They are mixed with a matrix of small organic molecules presenting strong absorption of the laser wavelength. The matrix compound, usually weak organic acids, must have high electronic absorption for the used laser wavelength, stable under vacuum, low vapour pressure, good solubility in solvents that dissolve the analyte, and good miscibility with the analyte in the solid state. Examples of commonly used matrices for synthetic polymers are 2,5- dihydroxybenzoic acid, dithranol (1,8,9-trihydroxyanthracene (DHB), 3- $\beta$-indoleacrylc acid (IAA), and 2,(-4hydroxyphenylazo)benzoic acid (HABA) $[9,10]$.

In this study, PETG was dissolved in phenol/chloroform, 1/1 (v/v), and DHB was selected for the matrix. A dried droplet sample was prepared by putting $1 \mu \mathrm{L}$ of polymer in phenol/chloroform, $1 / 1(\mathrm{v} / \mathrm{v})$, and $1 \mu \mathrm{L}$ of a mixture of $1 \mathrm{ml}$ of DHB in phenol/chloroform, $1 / 1$ $(\mathrm{v} / \mathrm{v})$, as a matrix on the target plate. The test was conducted using the Axima Confidence mass spectrometer Plus MALDI-TOF/TOFMS analyser (Shimadzu Biotech, Japan) equipped with a Nitrogen laser emitting at $337.1 \mathrm{~nm}$ allowing data acquisition in positive ion mode. The acceleration voltage used was $20 \mathrm{KV}$ and the extraction delay time used was $15000 \mathrm{in}$ reflector MS mode. Data acquisition and data processing were carried out using the Kratos analyser software (Kratos, UK). All mass spectra were collected by averaging the signal of 1620 laser shots.

\subsection{Mechanical characterisation}

Uniaxial tensile tests were carried out using the SAUTER TVL machine (Sauter GmbH, UK) in dry state with a $500 \mathrm{~N}$ load cell and two AC 01R fine point clamps fitted to the platform and the loadcell. Five tensile samples were tested up to $\sim 2 \%$ strain which is the yield point.

\subsection{Shape recovery}

Shape recovery was evaluated by heating the printed structure above the glass transition temperature $\left(\mathrm{T}_{\mathrm{g}}\right)$, apply a force to deform the structure, cooling down the structure below $\mathrm{T}_{\mathrm{g}}$ to keep its temporary shape, remove the force, and finally heating up the structure again over $\mathrm{T}_{\mathrm{g}}$ allowing the structure to regain its permanent shape [11]. This procedure was repeated considering 5 and 10 cycles to investigate the effect of the cycle number on the shape recovery process. The steps followed to deform the structure and keeping its temporary shape is considered as programming and the following step of reheating the structure to regain its permanent shape is considered as the recovery. The PETG has a glass transition temperature of $75^{\circ} \mathrm{C}$, and both programming and recovery were carried out at $80^{\circ} \mathrm{C}$ [12]. 


\section{Results and discussion}

\subsection{Chemical analysis}

The MALDI-TOF MS spectrum of PETG is presented in Figure 2 showing a broad peak in the high mass range, and consequently the structural information cannot be obtained. Results show that PETG has a TPA backbone which is common in other polyesters with different diols which produce different properties. It is also possible to observe the presence of CHDM (not present in PET), which improves the printability of PETG and reduces its crystallinity $[9,10]$. In Figure 2 two lines were drawn, a black line corresponding TPA+EG and a blue line corresponding to TPA+CHDM $[13,14]$. Besides the TPA+EG and TPA+CHDM peaks the MALDI spectrum contains other peaks that correspond to fragment ions.

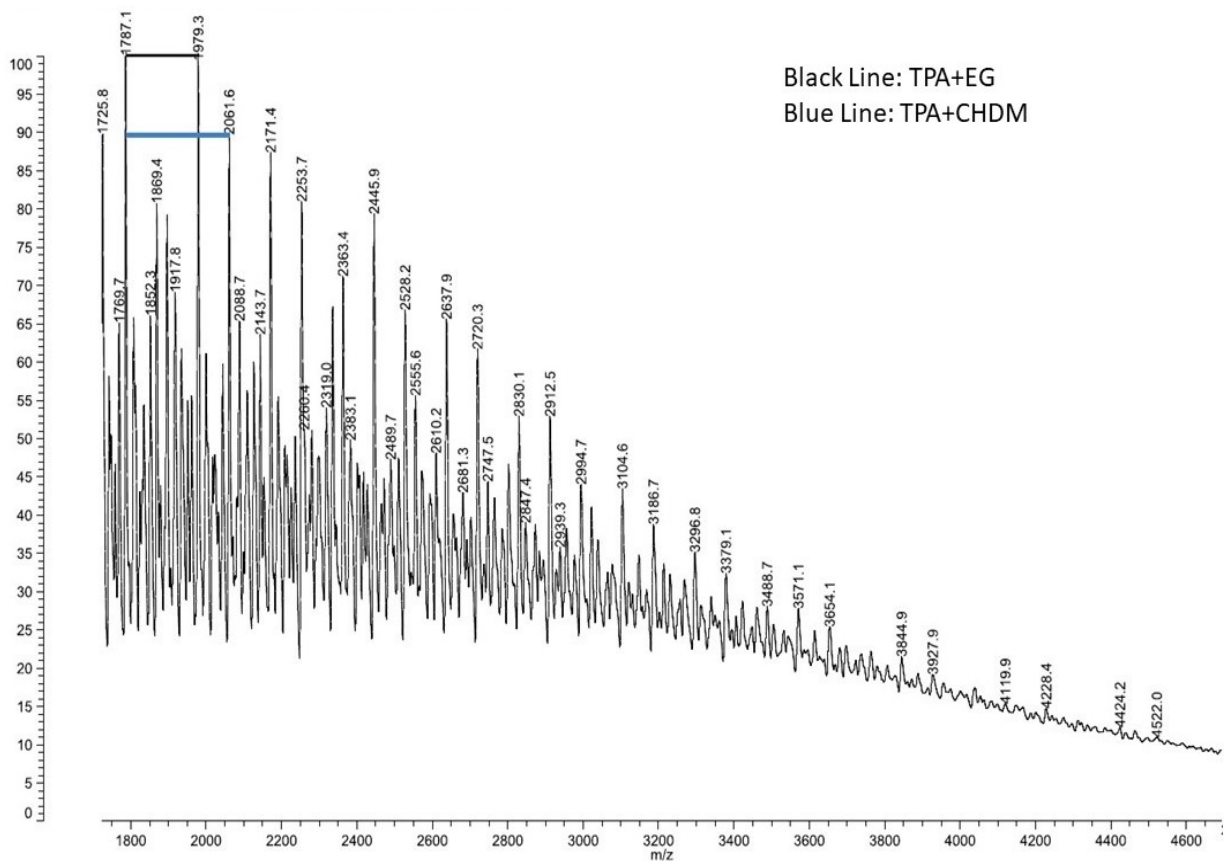

Fig 2. PETG MALDI-TOF MS spectrum where the $\mathrm{Y}$-axis is relative abundance and $\mathrm{X}$-axis is the mass per charge considered as mass in mass spectrometry

Table 2 highlights the constituent compounds that make up PETG. Molecular weight results were determined considering the condensation reaction that produces the oligomers.

Table 2. PETG Chemical components

\begin{tabular}{lll}
\hline Compound & Molecular Formula & Molecular weight \\
\hline Terephthalic acid (TPA) & $\mathrm{C}_{8} \mathrm{H}_{6} \mathrm{O}_{4}$ & 166.13 \\
Ethylene Glycol (EG) & $\mathrm{CH}_{2} \mathrm{OH}_{2}$ & 62.07 \\
Cyclohexanedimethanol (CHDM) & $\mathrm{C}_{8} \mathrm{H}_{16} \mathrm{O}_{2}$ & 144.21 \\
\hline
\end{tabular}




\subsection{Mechanical Tensile test}

The mechanical tensile test results obtained for the five tested samples are presented in Table 3. As observed the overall elastic modulus is around $1756 \mathrm{MPa}$, lower to PET due to the reduced crystallinity level, but higher than other polymers commonly used for bone scaffolds such as PCL, and PLA $[15,16]$.

Table 3. Mechanical properties of PETG

\begin{tabular}{llcccc}
\hline Test & Force $(\mathrm{N})$ & Displacement $(\mathrm{mm})$ & Stress $(\mathrm{MPa})$ & Strain & Modulus $(\mathrm{MPa})$ \\
\hline Test 1 & 399.70 & 1.53 & 49.94 & 0.02 & 1749.41 \\
Test 2 & 404.30 & 1.56 & 42.42 & 0.02 & 1726.87 \\
Test 3 & 397.50 & 1.58 & 41.71 & 0.02 & 1676.34 \\
Test 4 & 404.90 & 1.46 & 42.49 & 0.02 & 1847.89 \\
Test 5 & 400.10 & 1.49 & 41.98 & 0.02 & 1789.21 \\
Average & 401.30 & 1.52 & 42.11 & 0.02 & 1756.20 \\
\hline
\end{tabular}

\subsection{Shape recovery results}

Figure 3 shows the shape recovery of a PETG printed structure in response to heat changes. Figure $3(\mathrm{~A})$ shows the original printed structure before any programming or recovery takes place, Figure 3(B) shows the recovered shape after one cycle of programming and recovery, Figure $3(\mathrm{C})$ shows the recovered shape after five cycles of programming and recovery, and Figure 3(D) shows the recovered shape after ten cycles of programming and recovery. All cycles were given a period of 10 s to recover.

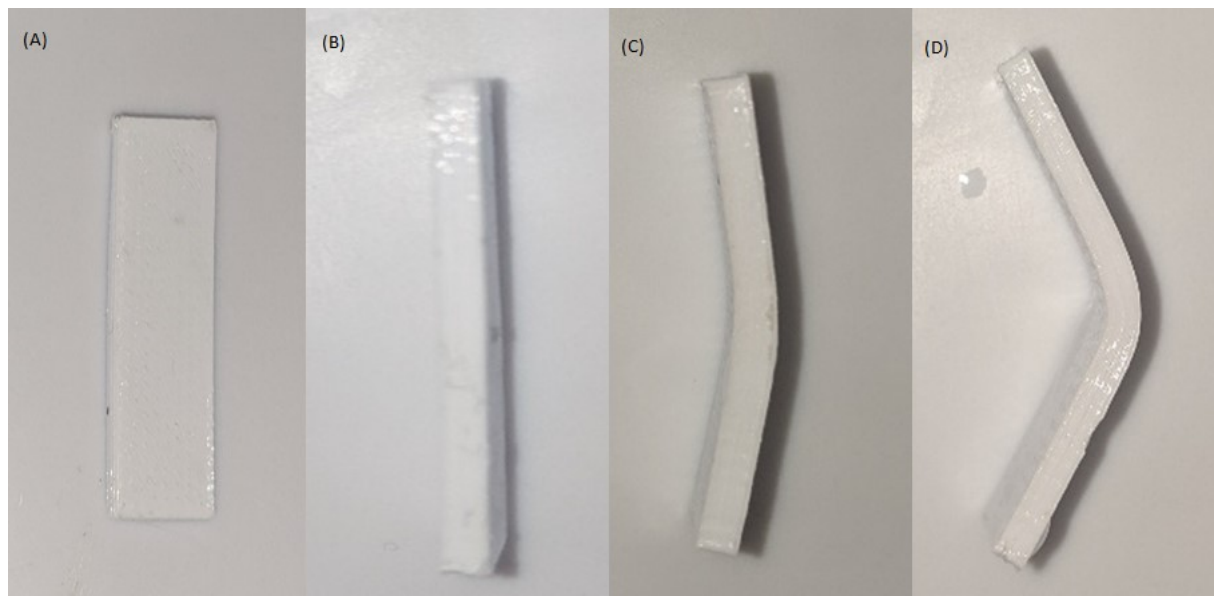

Fig 3. Shaper recovery for PETG using different recovery cycles, (A) original printed structure, (B) shape recovered after 1 cycle, (C)shape recovered after 5 cycles, (D) shape recovered after 10 cycles.

The crystalline and amorphous phases in SMPs determine the shape memory behaviour. The amorphous phase is related to shape fixity and the crystalline phase is related to shape recovery. As previously reported, PETG has a 70\%: 30\% ratio of amorphous to crystalline 
phases which explains the deteriorating shape recovery performance as the number of cycles increase $[17,18]$.

\section{Conclusion}

This paper shows that PETG is a suitable material for 4D Printing applications. In the case of bone tissue engineering applications, PETG exhibit higher mechanical properties than commonly used polymers such as PCL and PLA $[15,16]$. Maldi-ToF MS confirmed the presence of CHDM that is responsible for decreasing PET crystallinity, hence increasing its printability. Further investigation needs to be carried out on PETG considering different factors such as morphological changes with time, potential shape change upon other external stimuli rather than temperature, degradability and biological behaviour.

\section{Reference:}

1. J. Kim, Biopolymer Composites in Electronics, p. 311-331 (2017)

2. Z. Zhang, K.G. Demir, and G.X. Gu, Int J Smart Nano Mater,10(3): p. 205-224 (2019)

3. M. Bashir, C.F. Lee, and P. Rajendran,J Eng Appl Sci, 12: p. 50-56 (2017)

4. C.N.Bowman, Polymer,23(55): p. 5847-5848 (2014)

5. P. Bártolo, and B. Bidanda, Bio-materials and prototyping applications in medicine,p. 15-26 (2008)

6. R. Dwivedi, S. Kumar, R. Pandey, A.Mahajan, D. Nandana, D.S. Katti, and D. Mehrotra,J.Oral Biol Craniofac Res.10(1),9.381-386 (2020)

7. C. Muehlendfeld, S.A. Roberts , 3D and 4D printing in biomedical applications: process engineering and additive manufacturing,pp. 1-23 (2019)

8. E. Pei,and G.H. Loh, Technological considerations for 4D printing: an overview. Prog. in Addit. Manuf.,3(1-2): p. 95-107 (2018)

9. G. Montaudo, F. Samperi, and M. Montaudo, Prog. in Poly. Sci. 31(3),p. 277-357 (2006)

10. T. Wu, H.L. Hu, INT J POLYM ANAL CH, 19(5): p. 441-452 (2014)

11. M. Heuchel, J. Cui, K. Kratz, H. Kosmella, A. Lendlein,Adv. in Poly. Sci. 51 (26),p.6212-6218 (2010)

12. B. Yang, W. Huang,C. Li, and L. Li , Poly., 47(4): p. 1348-1356 (2006)

13. S.Turner,J. of Poly. Sci. Part A: Poly. Ch.,42(23): p. 5847-5852 (2004)

14. L. Charles, Mass Spec. Rev.,33(6),p.523-543 (2013)

15. H.S. Abdo, A.A. Elzatahry, H.F. Alharbi, and K.A. Khalil, Biopolymer Composites in Electronics, p. 13-25 (2017)

16. G. Narayanan,V.N. Vernekar, L.E.Kuyinu, and T.C. Laurencin, Adv. Drug Deliv. Rev.,107, p. 247-276 (2016)

17. T. Chen,G. Jiang, G.Li, Z. Wu, and J. Zhang, RSC Advances, 5(74): p. 60570-60580 (2015)

18. S. Abdullah, A. Jumahat, N. Abdullah, and L. Frormann, Procedia Engineering, 41, p.1641-1646 (2012) 